\title{
Selbstreferenz als Gesetzgebungsprogramm?
}

- Zu einigen neueren Entwicklungen im Recht des Hochschul- und Justizwesens -

\section{Einleitung: Selbstreferenz und Selbstregulierung}

Kaum zu Unrecht ist vor einigen Jahren betont worden ${ }^{1}$, daß weite Teile des rechtswissenschaftlichen Diskurses, insbesondere jene, die sich selbst »Innovationsoffenheit $\aleph^{2}$ auf die Fahnen geschrieben haben ${ }^{3}$, in ihrer den im engeren Sinne juristischen Debatten zugrunde gelegten Analyse der gegenwärtigen Gesellschaftsverhältnisse in starkem Maße auf Gedanken der Systemtheorie ${ }^{4}$ rekurrieren. Insbesondere die Figur der Ausdifferenzierung der Sozialsphären, die den modernen Gegenpol zur früher gegebenen stratifizierten, also statischen, hierarchisch organisierten Gesellschaft benennen soll ${ }^{5}$, sowie der Gedanke der Selbstreferentialität der solcherart sich bildenden Teilsysteme scheinen fast zu einer Art common sense der Debatte geworden zu sein ${ }^{6}$. Selbstreferenz im systemtheoretischen Sinne meint dabei bekanntlich die Abgeschlossenheit einer spezifischen sozialen Sphäre, die sich dadurch auszeichnet, daß ihre internen Operationen immer nur an eigene Prozesse anschließen und alles, was sich dem

1 Vgl. O. Lepsius, Steuerungsdiskussion, Systemtheorie und Parlamentarismuskritik, Tübingen 1999.

2 Zum Begriff vgl. W. Hoffmann-Riem, Innovationsoffenheit und Innovationsverantwortung durch Recht. Aufgaben rechtswissenschaftlicher Innovationsforschung, AöR 131 (2006), S. $255 \mathrm{ff}$.

3 Vgl. hierzu die Beiträge in der von W. Hoffmann-Riem, K.-H. Ladeur und H.-H. Trute herausgegebenen Reihe »Schriften zur rechtswissenschaftlichen Innovationsforschung «, Baden-Baden 1998ff., insbes. Bd. 1: W. Hoffmann-Riem/J.-P. Schneider (Hrsg.), Rechtswissenschaftliche Innovationsforschung. Grundlagen, Forschungsansätze, Gegenstandsbereiche, Baden-Baden 1998.

4 »Systemtheorie« meint dabei v.a.: die Theorie Niklas Luhmanns. Hans Ulrich Gumbrecht (Über Niklas Luhmanns intellektuelles Vermächtnis, Merkur 688 (2006), S. 696ff., hier S. 706) hat zu Recht darauf hingewiesen, daß Luhmann zwar selbst stets auf »die Systemtheorie« wie auf eine als bekannt vorauszusetzende weltumspannende Forschungsbewegung rekurriert, es sich dabei jedoch - bis die ersten Adepten nachfolgten - weitgehend um eine one-man-show handelte.

5 Vgl. etwa N. Luhmann, Soziale Systeme. Grundriß einer allgemeinen Theorie, 4. Aufl. Frankfurt a.M. 1994, S. 624ff.; ferner die Beiträge in ders. (Hrsg.), Soziale Differenzierung. Zur Geschichte einer Idee, Opladen 1985.

6 Vgl. zur Ausdifferenzierung etwa R. Pitschas, Verwaltungsverantwortung und Verwaltungsverfahren. Strukturprobleme, Funktionsbedingungen und Entwicklungsperspektiven eines konsensualen Verwaltungsrechts, München 1990; D. Grimm, Die Zukunft der Verfassung, Frankfurt a.M., 2. Aufl. 1994, S. 402f.; U. Di Fabio, Das Recht offener Staaten. Grundlinien einer Staats- und Rechtstheorie, Tübingen 1998, S. 15ff.; zur Selbstreferentialität etwa M. Fehling, Neue Herausforderungen an die Selbstverwaltung in Hochschule und Wissenschaft, Die Verwaltung 35 (2002), S. 399ff., hier S. 400. 
eigenen Beobachtungsmuster nicht fügt, entweder als noise, als bloßes Störgeräusch, ignorieren, oder aber auf dem Wege des re-entry, in der von den eigenen Strukturen ausgehenden eingrenzenden Abgrenzung, re-integrieren ${ }^{7}$. Innerhalb des Sozialsystems Wirtschaft etwa ist demnach die Rechtmäßigkeit einer Handlung nur insofern von Bedeutung, als das Primärinteresse der Gewinnmaximierung durch interferierende Akte aus der Justizsphäre gestört werden könnte. Die intersystemische Einwirkung erfolgt nur mittelbar ${ }^{8}$. Systemintern dagegen bewirkt die so verstandene Autoreferentialität der Konzeption gemäß eine Selbststabilisierung, die aber zugleich nicht als Reduktion, sondern Multiplikation der Anschlußmöglichkeiten für die eigenen Kommunikationsprozesse fungieren soll, weil gegenüber einer paralysierenden Fülle von Möglichkeiten erst Selektion und Variation hinreichend konkrete Anknüpfungspunkte schaffen ${ }^{9}$.

In der innovationsfreundlichen rechtswissenschaftlichen Diskussion soll diese als sozialwissenschaftliches Theorem übernommene Rekursivität der Gesellschaftsbereiche nun dazu dienen, ein mit der Übernahme der systemtheoretischen Grundannahmen einhergehendes Problem durch diese selbst wieder zu kurieren, also gewissermaßen den Teufel mit dem Beelzebub auszutreiben. Weil vor dem Hintergrund der angenommenen sozialen Differenzierung der Gesellschaft eine direkte staatliche Steuerung der einzelnen Teilbereiche nur noch wenig erfolgversprechend erscheint, insbesondere das traditionelle Haupthandlungsinstrument des politischen Systems, das Gesetz, in seiner Wirkungskraft angezweifelt wird ${ }^{10}$, soll der gewünschte Effekt nunmehr mittelbar erzielt werden. In einer juridischen Variante dessen, was Odo Marquard für den Bereich der Philosophie mit dem schönen Ausdruck der »Inkompetenzkompensationskompetenz « bezeichnet hat ${ }^{11}$, wird nicht das konstatierte Steuerungsdefizit affirmativ gefaßt, als Anzeichen dafür, wovon der regulierende »Staat«, d.h. die Politik, besser die Finger lassen sollte, sondern ein spezifischer Trick vorgeschlagen, wie die fehlende Regelungskompetenz kompetent kompensiert werden könnte: durch Subdelegation. »Regulierte Selbstregulierung « ist das Zauberwort, das offenbar das perpetuum mobile eines die politischen Ziele verwirklichenden, gleichwohl den Eingriff mit geringstmöglicher Intensität vollziehenden, die Eigenrationalität des gesteuerten Bereichs ausnutzenden

7 Vgl. etwa die knappe Zusammenfassung in N. Luhmann, Das Recht der Gesellschaft, Frankfurt a.M. 1993, S. 76ff.; zu den Besonderheiten bzgl. des Rechts ferner ders., Closure and Openness: On Reality in the World of Law, in: G. Teubner (Hrsg.), Autopoietic Law: A New Approach to Law and Society, Berlin/New York 1988, S. 335ff.; ferner G. Teubner, Recht als autopoietisches System, Frankfurt a.M. 1989, S. $21 \mathrm{ff}$.

8 Zudem etablieren sich jedoch auch dauerhafte Kooperationsformen in Gestalt struktureller Kopplungen zwischen den Systemen; vgl. dazu N. Luhmann, Das Recht der Gesellschaft, S. 440ff.

9 Vgl. zum Problem allg. B. Waldenfels, Ordnung im Zwielicht, Frankfurt a.M. 1985, S. 51ff.

10 Vgl. dazu die Beiträge in dem fast kanonisch gewordenen Band D. Grimm (Hrsg.), Wachsende Staatsaufgaben - sinkende Steuerungsfähigkeit des Rechts, Baden-Baden 1990, dort insbesondere E.-H. Ritter, Das Recht als Steuerungsmedium im kooperativen Staat, a.a.O., S. 69ff. Skepsis demgegenüber bei E. Schmidt-Aßmann, Das allgemeine Verwaltungsrecht als Ordnungsidee. Grundlagen und Aufgaben der verwaltungsrechtlichen Systembildung, Berlin u.a. 1998, S. 19ff.

11 Vgl. O. Marquard, Abschied vom Prinzipiellen, Stuttgart 1981, S. $27 \mathrm{ff}$. 
politischen Handelns benennen soll ${ }^{12}$. Hierzu liegt mittlerweile bereits eine Reihe sektorenspezifischer Einzeluntersuchungen vor ${ }^{13}$. Parallel zu jenem Trend verläuft eine Entwicklung, der zufolge die gesetzlichen Regelungen verstärkt von der konkrete Handlungsanweisungen beinhaltenden traditionell konditionalen auf eine offenere finale Programmierung ${ }^{14}$ umgestellt werden ${ }^{15}$. Der »Gewährleistungsstaat ${ }^{16}$ entkoppelt Aufgabenwahrnehmung und Erfüllungsverantwortung ${ }^{17}$. Stärker formalisiert ausgedrückt: Statt der (Rechts-)Produzenten rückt das Produkt, also das Regelungsergebnis, in den Fokus der Aufmerksamkeit ${ }^{18}$. Der Frage, ob dadurch nicht Zuständig- und da-

12 Vgl. dazu die Beiträge in R. Mayntz/F. W. Scharpf (Hrsg.), Gesellschaftliche Selbstregelung und politische Steuerung, Frankfurt a.M. 1995, sowie jene in DV Beiheft 4 (2001): Regulierte Selbstregulierung als Steuerungskonzept des Gewährleistungsstaates, dort insbesondere K.-H. Ladeur, Die Regulierung der Selbstregulierung und die Herausbildung einer »Logik der Netzwerke«, a.a.O., S. 59 ff.; ferner U. Di Fabio, Verwaltung und Verwaltungsrecht zwischen gesellschaftlicher Selbstregulierung und staatlicher Steuerung, in: VVDStRL 56 (1997), S. 235ff. Dabei können näherhin verschiedene Stufen oder Typen der Aufgabenteilung bzw. des rechtlichen Steuerungsverfahrens - von anreizorientiert bis imperativisch - unterschieden werden; vgl. dazu etwa W. Hoffmann-Riem, Innovationsoffenheit und Innovationsverantwortung durch Recht, a.a.O., S. 275f.; ferner E. SchmidtAßmann, Zur Reform des Allgemeinen Verwaltungsrechts - Reformbedarf und Reformansätze -, in: W. Hoffmann-Riem/ders./G.F. Schuppert (Hrsg.), Reform des Allgemeinen Verwaltungsrechts. Grundfragen, Baden-Baden 1993, S. 11ff., hier S. 43f.

13 Vgl. etwa A. Finckh, Regulierte Selbstregulierung im Dualen System, Baden-Baden 1998; J.-P. Schneider, Liberalisierung der Stromwirtschaft durch regulative Marktorganisation, Baden-Baden 1999; W. Wegmann, Regulierte Marktöffnung in der Telekommunikation, Baden-Baden 2001; J. Junker, Gewährleistungsaufsicht über Wertpapierdienstleistungsunternehmen, Berlin 2003.

14 Zur Unterscheidung R. Breuer, Konditionale und finale Rechtssetzung, AöR 127 (2002), S. 523ff.; allg. N. Luhmann, Lob der Routine, in: ders., Politische Planung: Aufsätze zur Soziologie von Politik und Verwaltung, Opladen 1971, S. 113ff.; sehr kritisch ggü. Zweckprogrammen im Recht ders., Das Recht der Gesellschaft, a.a.O., S. 195ff.

15 Vgl. W. Hoffmann-Riem, Gesetz und Gesetzesvorbehalt im Umbruch. Zur Qualitätsgewährleistung durch Normen, AöR 130 (2005), S. 5ff., hier S. 15. Prägnanterweise läßt sich noch dieses so moderne Programm als säkularisierte Umsetzung eines Theologumenons im Schmittschen Sinne lesen: Im Unterschied zur griechischen Kosmogonie »hat der biblische Schöpfungsgedanke nicht demiurgische, sondern imperative Orientierung. Der Herr befiehlt, und es geschieht. Er muß sagen, was geschehen soll, aber nicht, wie es geschehen soll. [...] Dem gebietenden Gestus entspricht, daß der Schöpfer hinterdrein nachsieht, ob der Befehl befolgt wurde und wie gut alles geworden ist.« (H. Blumenberg, Die Lesbarkeit der Welt, 3. Aufl. Frankfurt a.M. 1996, S. 22f.)

16 Zum Begriff allg. M. Eifert, Grundversorgung mit Telekommunikationsleistungen im Gewährleistungsstaat, Baden-Baden 1998, v.a. S. 18ff., 139ff.; vgl. ferner K-.H. Ladeur/T. Gostomzyk, Der Gesetzesvorbehalt im Gewährleistungsstaat, Die Verwaltung 36 (2003), S. 141ff.; G.F. Schuppert, Verwaltungswissenschaft, Baden-Baden 2000, S. 933ff.; W. Hoffmann-Riem, Das Recht des Gewährleistungsstaates, in: G.F. Schuppert (Hrsg.), Der Gewährleistungsstaat - ein Leitbild auf dem Prüfstand, Baden-Baden 2005, S. 87ff.

17 Vgl. W. Hoffmann-Riem, Gewährleistungsstaat und Gewährleistungsrechtsprechung - am Beispiel regulierter Selbstregulierung (FS-Beitrag, i.E.).

18 Vgl. zu dieser Produktfixierung mit Blick auf das sog. »Neue Steuerungsmodell« M. Eifert, Das Neue Steuerungsmodell - Modell für die Modernisierung der Gerichtsverwaltung?, Die Verwaltung 30 (1997), S. 75ff.; daran anknüpfend A. Voßkuhle, Das >Produkt< der Justiz, in: H. Schulze-Fielitz/C. Schütz (Hrsg.), Justiz und Justizverwaltung zwischen Ökonomisierungsdruck und Unabhängigkeit, DV Beiheft 2002, S. $35 f f$. 
mit Verantwortlichkeiten, auf deren genauer Fixierung das überkommene hierarchische System doch beruhte, um in letzter Konsequenz das demokratische Votum über das Getane zu gewährleisten, in bedenklichem Maße verunklart würden ${ }^{19}$, wird durch den Hinweis begegnet, die Verantwortlichkeit werde nicht so sehr delegiert als vielmehr modifiziert: von einer institutionell verstandenen zur eher sachlich geprägten ${ }^{20}$. Diese modifizierende Verschiebung mag indes wiederum Zweifel bezüglich der Transparenz des Modells hervorrufen. Selbst wenn man solche Bedenken aber zurückstellte und die demokratische Komponente als nicht entscheidend einordnete, wenn man die Ausdifferenzierungsthese als plausibel hinnähme und sich nicht in eine schematische Parteinahme nach dem Muster Systemtheorie hui, Deliberation pfui (oder umgekehrt) verwickeln ließe $\mathrm{e}^{21}$, ist dennoch auch in einer immanent bleibenden Perspektive, die das Konzept primär an seinen eigenen Ansprüchen mißt $\mathrm{t}^{22}$, weiter zu fragen, ob eine derartige Delegation von Verantwortung auf die einzelnen Sozialbereiche nicht ihrerseits zu Friktionen führen könnte, die statt der Vervielfältigung der internen Anschlußmöglichkeiten nur Selbstblockaden hervorrufen dürfte. In Anknüpfung an die (von Lepsius in normativer Hinsicht allerdings bereits als Vorwurf formulierte) These, die Ausgangsposition der Protagonisten der Steuerungsdiskussion habe sich »von der Rechtmäßigkeit und Rechtsförmlichkeit auf die Sachangemessenheit und die Effektivität verschoben ${ }^{23}$, ist insofern zu untersuchen, wie es um diese Effektivität $d e$ facto bestellt ist: Die konsequenzbelastete Übertragung von Verantwortlichkeit für das eigene Handeln bedingt Selbstüberwachung, also eine Aufwendung eines Teils der eigenen Energie nicht für die produktive Kerntätigkeit, sondern Supervision. Die perfektionierte Kontrolle aber mündet in Paralyse: Risikominimierung im Bereich etwa des Produkthaftungsrechts ist einerseits natürlich erforderlich, weil den Staat eine verfassungsrechtlich gebotene Schutzpflicht hinsichtlich insbesondere der körperlichen Unversehrtheit seiner Bürger trifft ${ }^{24}$, die allein durch retrospektive Kompensationsverfahren, wie sie insbesondere das Zivilrecht zur Verfügung stellt, nicht hinreichend erfüllt

19 Kritisch aus normativer Sicht insofern O. Lepsius, Steuerungsdiskussion, Systemtheorie und Parlamentarismuskritik, a.a.O., S. 14ff.

20 Vgl. dazu Chr. Möllers, Braucht das öffentliche Recht einen neuen Methoden- und Richtungsstreit? VerwArch 90 (1999), S. 187ff., hier S. 201: »Die Legitimationsleistung wird vom Erfordernis staatlicher Entscheidung zum Erfordernis staatlicher Verantwortung der richtigen Entscheidung verschoben.« Möllers spricht in diesem Zusammenhang von »expertokratische[n] Rechtfertigungsmodellen«. Zu weiteren Differenzierungen vgl. S. Augsberg, Rechtsetzung zwischen Staat und Gesellschaft. Möglichkeiten differenzierter Steuerung des Kapitalmarktes, Berlin 2003, S. 75 m.w.N.

21 Zur Habermas'schen Skepsis gegenüber der Ausdifferenzierungsthese vgl. kritisch N. Bolz, Am Ende der Gutenberg-Galaxis. Die neuen Kommunikationsverhältnisse. München, 2. Aufl. 1995, S. 28ff.

22 Zum theoretischen Modell eines solchen Verfahrens immanenter Kritik (und seinen Grenzen) vgl. Th. W. Adorno, Prismen, in: ders., Ges. Schr. Bd. 10.1, Frankfurt a.M. 1977, S. 27f.; ders., Minima Moralia, Ges. Schr. Bd. 4, 2. Aufl. Frankfurt a.M. 1996, S. 171f.

23 O. Lepsius, Steuerungsdiskussion, Systemtheorie und Parlamentarismuskritik, a.a.O., S. 3f.

24 Vgl. dazu jüngst etwa $C$. Callies, Die grundrechtliche Schutzpflicht im mehrpoligen Verfassungsrechtsverhältnis, JZ 2006, S. $321 \mathrm{ff}$. 
werden $\mathrm{kann}^{25}$, muß andererseits jedoch auch Grenzen finden, weil ansonsten jede Innovation, die als Neuerung eo ipso riskant, da noch unbekannt ist, ausgeschlossen würde. Insofern entsprach es, wie Karl-Heinz Ladeur herausgestellt hat, der Konzeption des liberalen Rechtsstaats, gegenüber einer fortschreitenden, ausufernden Zuschreibung von Verantwortlichkeiten spezifische Stopp-Regeln zu etablieren und dergestalt für eine gewisse Entlastung der Handelnden zu sorgen ${ }^{26}$. Weil das mit Innovationen zwangsläufig verbundene Risiko (daß Handeln sich schuldig machen heißt und der Handelnde daher immer gewissenlos sein muß, ist spätestens seit dem 18. Jahrhundert ein auch literarisch verhandelter Topos ${ }^{27}$ ) für die Gesellschaftsentwicklung insgesamt produktiv war, durfte nicht der einzelne Erfinder oder Konstrukteur allein mit deren etwaigen Problemen und Kosten belastet werden. Die Entlastung wurde dann auf einer anderen, überindividuellen Ebene wieder aufgefangen, insbesondere durch die Etablierung eines Versicherungswesen ${ }^{28}$. Gegenüber einer solchen Externalisierung von Folgenverantwortung bildet das Selbstregulierungs-Modell in gewissem Sinne die Antithese: Ansätze zur Problembewältigung werden nun nicht mehr verschoben und an externe Bearbeiter delegiert, sondern im Anschluß an die internen Prozesse gesucht. $\mathrm{Da} \beta$ ein solches partizipatorisches Verfahren hinsichtlich der Problemnähe und der damit verbundenen Sachkompetenz der Entscheidungsträger Vorzüge hat, ist evident ${ }^{29}$. Ebenso deutlich sind aber auch die Schwierigkeiten des Modells: Wo die eigene Hauptaufgabe zunehmend darin liegt, die eigene zuvorige Hauptaufgabe zu kontrollieren, tritt das System in den Zustand eines negativen Narzißmus' ein, in dem es nicht selbstverliebt, sondern höchst mißtrauisch, voll grimmigen Verdachts, sein Ebenbild beschaut. Selbstregulierung ist insofern nicht bloß ein soziologisch plausibles Konzept, sondern auch eine Gefahr.

$\mathrm{Da} ß$ diese Gefahr nicht nur theoretischer, sondern höchst praktischer Natur ist, läßt sich mittlerweile an Beispielen studieren. Denn in jüngerer Zeit hat offenbar die Gesetzgebung die Idee der Selbstregulierung für sich entdeckt. Dabei kann hier zunächst dahinstehen, ob damit tatsächlich theoretische Implikationen umgesetzt werden sollten oder vielmehr schlichte Sparzwänge handlungsmotivierend wirkten. Entscheidend ist das Ergebnis: Sowohl durch die von allen Bundesländern, unabhängig von der parteipolitischen Orientierung der jeweiligen Regierung, beharrlich betriebene »Hochschulmodernisierung « als auch durch das jüngst vorgestellte Konzept einer »Untätigkeitsbeschwerde«

25 Vgl. K.-H. Ladeur, Die rechtliche Steuerung von Entwicklungsrisiken zwischen zivilrechtlicher Produkthaftung und administrativer Sicherheitskontrolle, BB 1993, S. 130ff.

26 Vgl. K.-H. Ladeur, Negative Freiheitsrechte und gesellschaftliche Selbstorganisation. Die Erzeugung von Sozialkapital durch Institutionen, Tübingen 2000, S. 56ff.

27 Vgl. etwa J.W. v. Goethe, Die Leiden des jungen Werther, in: Goethes Werke (Hamburger Ausgabe), München 1998, Bd. VI, S. 52f.; ders., Maximen und Reflexionen, in: Goethes Werke, a.a.O., Bd. XII, Nr. 251, S. 399.

$28 \mathrm{Vgl}$. K.-H. Ladeur, Negative Freiheitsrechte und gesellschaftliche Selbstorganisation, a.a.O., S. 100f., 262f.; allg. zur Entwicklung der Versicherungsmentalität P. Sloterdijk, Im Weltinnenraum des Kapitals. Für eine philosophische Theorie der Globalisierung, Frankfurt a.M. 2005, S. 147ff.

29 Vgl. allg. Th. Groß, Grundzüge der organisationswissenschaftlichen Diskussion, in: E. Schmidt-Aßmann/W. Hoffmann-Riem (Hrsg.), Verwaltungsorganisationsrecht als Steuerungsressource, Baden-Baden 1997, S. 139ff., hier S. 142f. 
im Bereich der Justiz wird Selbstüberwachung zur Pflichtaufgabe erklärt ${ }^{30}$. Während dort unter dem allgemeinen Schlagwort von der »Stärkung der Hochschulautonomie« u.a. die Qualität von Forschung und Lehre dadurch gewährleistet werden soll, daß Wissenschaftler einen Teil ihrer Zeit zur Bewertung der Leistung der Kollegen aufwenden, wird hier offenbar sektorspezifisch eingeführt, was Art. 19 Abs. 4 GG nach der herrschenden Auffassung bislang noch nicht gewährte: ein Anspruch auf richterliche Kontrolle der Gerichte $^{31}$. Bemerkenswert ist diese Entwicklung insbesondere vor dem Hintergrund, daß zeitgleich interne Prüfungen in anderen Bereichen als ineffizient beseitigt werden sollen: So wurde in Niedersachsen die weitgehende Abschaffung des verwaltungsrechtlichen Widerspruchsverfahrens beschlossen, da dieses in der weit überwiegenden Anzahl der Fälle den sich anschließenden Rechtsstreit vor den Gerichten doch nicht verhindern konnte $^{32}$. Gleichwohl wird vom selben Land die Hochschulreform samt interner Evaluationsverpflichtungen mit ungebremstem Eifer vorangetrieben. Eine strukturell vergleichbare Problematik besteht aus Sicht der niedersächsischen Landesregierung offenbar nicht.

\section{Die Hochschulreform}

Nachdem mit der HRG-Novellierung aus dem Jahr 1998 die bisherigen bundesgesetzlichen Vorgaben bezüglich der Hochschulorganisation gem. §§ 61-66 HRG a.F. ${ }^{33}$ ersatzlos gestrichen wurden, eröffnete sich ein neues Betätigungsfeld für die Bundesländer, das zu einer umfassenden Modernisierung im Sinne einer »Umgestaltung der deutschen Hochschullandschaft« genutzt werden sollte ${ }^{34}$. Unter dem proklamierten allgemeinen Ziel einer Erweiterung der Autonomie der Hochschulen ${ }^{35}$ wurden im

$30 \mathrm{Zu}$ weiteren Beispielen einer Verabschiedung klassisch-hoheitlich ausgeübter Kontrollfunktionen zugunsten einer verstärkten Verlagerung auf private Fremd- und Eigenkontrollen vgl. instruktiv M. Eifert, Die geteilte Kontrolle. Die Beteiligung Privater an der Rechtsverwirklichung, Die Verwaltung 39 (2006), S. 309ff., v.a. S. $312 \mathrm{ff}$. Zu einer gewissen Trendumkehr im Bereich der Unternehmenskontrolle vgl. S. Augsberg, Verfassungsrechtliche Aspekte einer gesetzlichen Offenlegungspflicht für Vorstandsbezüge, ZRP 2005, S. 105ff.; ders., Vom Codex zur Kodifizierung, ZG 2005, S. $315 \mathrm{ff}$.

31 Vgl. BVerfGE 11, 263 (265); 76, 93 (98); BVerfG NJW 2003, S. 1924 (1925); H.-J. Papier, in: Isensee/Kirchhof, HdBStR VI, 2. Aufl. Heidelberg 2001, § 154, Rn. 37; eher befürwortend dagegen etwa E. Schmidt-Aßmann, in: Maunz/Dürig, GG (Stand: Febr. 2003), Art. 19 Abs. 4, Rn. 96ff.; ausführlich A. Voßkuhle, Rechtsschutz gegen den Richter, München 1993, v.a. S. 147ff., 255ff.

32 Vgl. dazu U. Rüssel, Zukunft des Widerspruchsverfahrens, NVwZ 2006, S. 523ff.

$33 \mathrm{Zu}$ den Organisationsgrundsätzen durch diese Bestimmungen vgl. für die frühere Rechtslage U. Karpen, in: Hailbronner/Geis, HRG-Kommentar, § 61, Rn. 1ff., $35 \mathrm{ff}$.

34 BR-Drucksache 724/97, 15. Zu den Umsetzungen in den Bundesländern s. G. Sandberger, Organisationsreformen und Autonomie - Bewertung der Reformen in den Ländern, WissenschaftsR 35 (2002), S. $125 \mathrm{ff}$.

35 Vgl. G. Sandberger, Organisationsreformen und Autonomie, a.a.O., S. 126; D. MüllerBöling, Die entfesselte Hochschule, Gütersloh 2000, S. 37ff.; E. Hödl/W. Zegelin, Hochschulreform und Hochschulmanagement, Marburg 1999, S. 131ff. Der Konflikt zwischen Auto- und Heteronomie der Universitäten ist natürlich alt; vgl. dazu etwa $R$. Brandt, Universität zwischen Selbst- und Fremdbestimmung. Kants >Streit der Fakultäten<. Berlin 2003 , v.a. S. 163ff., mit dem Hinweis, die heutige Bedrohung gehe primär nicht mehr von Kirche oder Staat, sondern der »kommerziellen Gesellschaft« aus. 
Zuge einer Verlagerung der früher durch den Staat vorgenommenen Steuerungstätigkeit insoweit vor allem in zwei Richtungen Veränderungen in Gang gesetzt. Zum einen soll die früher primär durch unmittelbar staatliche Stellen wahrgenommene Aufsicht über die Universitätstätigkeit nunmehr primär durch universitätsinterne, aber auch durch neu geschaffene intermediäre Organe gewährleistet werden. Der Großraum politischer Umgestaltung der Hochschullandschaft in dem Versuch einer Reform der universitären Organisationsstrukturen ist insofern, mit den Worten des Bundesverfassungsgerichts, »vor allem durch eine Stärkung der Leitungsorgane charakterisiert ${ }^{36}$. Zum Problem wird damit deren Zusammensetzung und organinterne Legitimation. Zum anderen soll die wissenschaftliche Leistung (und ebenso die Lehre) nicht mehr von auf Lebenszeit bestallten Professoren mehr oder minder unkontrolliert erbracht werden, sondern einer internen Supervision durch die Kollegen unterliegen. In beiden Varianten wurden demnach Regulierungs- und Kontrollaufgaben von staatlicher Seite delegiert.

\section{Stärkung der Leitungsorgane}

In einer Übertragung von Strukturen aus dem Wirtschaftsbereich - deren rechtliche Grundlagen vor allem die $\S \S 76 f f, \S \S 95 \mathrm{ff}$. AktG bilden - soll nach den Reformprojekten die universitätsinterne Leitung nun durch eine Art »Vorstand « erfolgen, dem flankierend ein »Aufsichtsrat «, bestehend aus größtenteils hochschulexternen Personen, an die Seite gestellt wird ${ }^{37}$. Dem liegt der Gedanke zugrunde, auch Wissenschaftsorganisation sei im Kern eine Managementaufgabe ${ }^{38}$. Ihre effektive und effiziente Bewältigung ist demzufolge vor allem dadurch zu gewährleisten, daß zum einen die Hochschulleitung in bezug auf ihren Personalbestand selbst professionalisiert wird und zum anderen zugleich erweiterte Befugnisse erhalten soll. Während die Professionalisierung etwa in der Verlängerung der hauptamtlich wahrzunehmenden Amtszeiten von Rektor und Prorektoren (sowie auf Fakultätsebene des Dekans) ${ }^{39}$ und die partielle Loskoppelung vom reinen Wissenschaftsbetrieb in der Wählbarkeit auch externer, Lei-

36 BVerfGE 111, 333 (334) (Brandenburgisches Hochschulgesetz); vgl. auch W. Kahl, Hochschulräte - Demokratieprinzip - Selbstverwaltung. Unter besonderer Berücksichtigung des Aufsichtsratsmodells in Baden-Württemberg, AöR 130 (2005), S. 225ff.

37 Vgl. J. Ipsen, Hochschulen als Unternehmen?, NdsVbl 2001, S. 6ff.; ders., Die neue niedersächsische Hochschulverfassung, NdsVBl 2002, S. 257ff.; M. Fehling, Neue Herausforderungen an die Selbstverwaltung in Hochschule und Wissenschaft, a.a.O., v.a. S. 416ff.; $G$. Püttner, Der Hochschulrat - oder: Was wird aus der deutschen Universität?, in: D. Doerr (Hrsg.), Die Macht des Geistes, FS für Hartmut Schiedermair 2001, S. 557ff.; K.F. Gärditz, Hochschulmanagement und Wissenschaftsadäquanz, NVwZ 2005, S. 407ff. Eine ausdrückliche - allerdings für die Universitäten noch fakultative - Übernahme der aktienrechtlichen Terminologie ist erfolgt in $\S 15$ Abs. 1 des 2004 novellierten BadenWürttembergischen Hochschulgesetzes.

$38 \mathrm{Vgl}$. zu den früheren Debatten, aber immer noch instruktiv (wenngleich mit dem speziellen Fokus auf außeruniversitäre Forschungseinrichtungen) $R$. Mayntz, Forschungsmanagement - Steuerungsversuche zwischen Scylla und Charybdis, Opladen 1985, v.a. S. 86ff.

39 Vgl. G. Sandberger, Organisationsreformen und Autonomie, a.a.O., S. 142, 148. 
tungserfahrung außerhalb der Hochschule aufweisender Kandidaten Ausdruck fin$\operatorname{det}^{40}$, zeigt sich die Aufgabenerweiterung v.a. in der Streichung der zuvor eingeräumten Allzuständigkeit des Repräsentativorgans Hochschulsenat ${ }^{41}$. Der Hochschulleitung kommt danach dem Konzept gemäß nunmehr nicht mehr nur eine rein operative, sondern auch eine strategische Gesamtverantwortung für die Struktur- und Entwicklungsplanung der Hochschule $\mathrm{zu}^{42}$. Bezüglich des Hauptsteuerungsinstruments zur Hochschulleitung erfolgte damit ein Paradigmenwechsel; die interne Aggregierung eines gemeinsamen Interesses der Körperschaftsmitglieder wurde ersetzt durch externen Fachverstand ${ }^{43}$, der sich durch seinen Erfolg in einem der ökonomischen Situation nachzubildenden Wettbewerb zwischen den Bildungs- und Forschungseinrichtungen legitimieren soll ${ }^{44}$. Dem Konzept liegt damit ein »Glaube an das Expertentum des Managers « zugrunde ${ }^{45}$.

Besonders drastisch zeigt sich dieser Paradigmenwechsel an einer etwas versteckten Stelle im Hamburgischen Hochschulgesetz (HmbHG) i.d.F. vom 27. Mai 2003. Dort findet sich eine Bestimmung, die das Verwaltungsrecht in dieser Form bislang nicht kannte. Denn bislang galt allgemein bezgl. der Satzungshoheit von mitgliedschaftlich verfaßten Selbstverwaltungseinheiten, daß diese Befugnis als ein »wesentliches Element der Selbstverwaltung ${ }^{46}{ }^{46}$ entweder von den Mitgliedern insgesamt oder aber, so in der Regel, von dem jeweiligen, eine spezifische demokratische Legitimation aufweisenden Hauptorgan der Körperschaft ausgeübt wird ${ }^{47}$. Prägnantes wie bekanntestes Beispiel dafür ist die Gemeinde; nach den Gemeindeordnungen der Länder zählt der Erlaß von Satzungen nicht nur zu den allgemeinen Aufgaben des Gemeinderats, sondern zu dessen Vorbehaltsaufgaben ${ }^{48}$. Diese dürfen nicht nur, sie müssen vom Gemeinderat ausgeübt werden; eine Delegation des Rechts, etwa an den Bürgermeister, ist un-

40 Vgl. G. Sandberger, Organisationsreformen und Autonomie, a.a.O., S. 142.

41 Vgl. etwa § 19 Abs. 1 UG BW; Art. 28 Bay HSchG; dazu G. Sandberger, Organisationsreformen und Autonomie, a.a.O., S. 145.

42 Vgl. G. Sandberger, Organisationsreformen und Autonomie, a.a.O., S. 140.

43 Vgl. dazu W. Kluth, Der Übergang von der selbstverwalteten Gruppenuniversität zur Hochschule als autonomer Forschungs- und Dienstleistungseinheit. Überblick und Analyse der unterschiedlichen Reformansätze in Landeshochschulgesetzen im Zeitraum 1998 bis 2004, RdJB 2004, S. 174ff., hier S. 187.

44 Vgl. K.-H. Ladeur, Die Wissenschaftsfreiheit der »entfesselten Hochschule«. Umgestaltung der Hochschulen nach Ermessen des Staates?, DÖV 2005, S. 753ff., hier S. 754f.

45 Vgl. kritisch dazu A. MacIntyre, Der Verlust der Tugend. Zur moralischen Krise der Gegenwart, Frankfurt 1987, S. 147, der den »Begriff der Effektivität des Managers« als eine »zeitgenössische moralische Fiktion« bezeichnet. Diesem Glauben korreliert die Konjunktur des Beratungswesens, das nun auch auf den öffentlichen Sektor ausgreift und sich seinerseits auf eine allgemein zu beobachtende »cultura terapeutica «, in der wir uns befinden, zurückführen läßt. Vgl. hierzu $P$. A. Rovatti, La filosofia può curare? La consulenza filosofica in questione, Mailand 2006, S. 17ff.

46 BVerfGE 12, 319 (325).

47 Vgl. H. Maurer, Allgemeines Verwaltungsrecht, 13. Aufl. München 2000, § 4, Rdnr. 15. F. Ossenbühl, Satzung, in: Isensee/Kirchhof, HStR III, 2. Aufl. Heidelberg 1996, § 66, Rdnr. 22 , spricht von »demokratisch legitimierten Gremien niederer Organisationsstufen«.

48 Vgl. etwa $§ 4$ GemO-BW; Art. 23 GO Bay; § 5 GO Bbg; § 5 GO Hess; § 7 GO NW; § 4 GO Schl-H. 
zulässig $^{49}$. Die grundlegenden, strukturprägenden Entscheidungen hinsichtlich der Bewältigung der (grund)gesetzlich der Körperschaft übertragenen Aufgaben dürfen nicht monokratisch erfolgen, sondern erfordern die Befassung jenes Organs, in dem die Interessen sämtlicher Körperschaftsmitglieder über deren Interessenvertreter wirksam aggregiert werden. Entsprechend wäre zu erwarten, daß auch die Satzungen der Selbstverwaltungskörperschaft Universität ${ }^{50}$ durch das die Körperschaftsmitglieder - gemäß $\S 36$ Abs. $1 \mathrm{HRG} »$ die an der Hochschule nicht nur vorübergehend oder gastweise hauptberuflich Tätigen, die eingeschriebenen Studierenden sowie die Doktorandinnen und Doktoranden « - repräsentierende Organ erlassen werden. Derartige Regelungen finden sich denn auch in den Hochschulgesetzen der Bundesländer ${ }^{51}$. Grds. gilt diese Regel auch für die Hamburger Hochschulen: Gemäß § 85 Abs. 1 Nr. 1 HmbHG ist der Hochschulsenat als das Vertretungsorgan der Körperschaftsmitglieder für die Beschlußfassung über die Grundordnung der Universität sowie über andere Satzungen zuständig. Von dieser Regel wird durch das novellierte Hamburger Gesetz aber eine signifikante Ausnahme normiert: die allgemeine Zuständigkeitsregelung gilt nur unter dem Vorbehalt, »soweit dieses Gesetz keine andere Zuständigkeit bestimmt« (§ 85 Abs. 1 Nr. 1 a.E. HmbHG). Eine derartige abweichende Zuständigkeitsregelung aber enthält § 79 Abs. 2 S. 2 HmbHG. Dieser bestimmt ausdrücklich, daß »Wirtschaftspläne und Gebührensatzungen « durch das Präsidium, dem gemäß $§ 79$ Abs. 1 HmbHG Präsident(in), Vizepräsident(in) und Kanzler(in) der Hochschule angehören, beschlossen werden. Die Satzungsbefugnis ist demnach in diesem Sachgebiet nicht dem zentralen Repräsentativ-, sondern dem Leitungsorgan zugewiesen ${ }^{52}$. Daß mit einer derartigen Erlaßform Legitimationsdefizite verbunden sein könnten, war dabei offenbar durchaus bewußt; zumindest lassen sich in dieser Hinsicht bestimmte Kompensationsbemühungen des Gesetzgebers verstehen. Anders als die durch den Hochschulsenat beschlossenen Satzungen bedürfen die durch die Hochschulleitung erlassenen Rechtsnormen noch einer zusätzlichen prozeduralen Absicherung, sie müssen eigens genehmigt werden ${ }^{53}$. Gemäß $\S 84$ Abs. 1 Nr. 7 HmbHG kommt dieses spezifische Recht zur »Geneh-

49 Vgl. nur E. Schmidt-Aßmann, Kommunalrecht, in: ders. (Hrsg.), Besonderes Verwaltungsrecht, 11. Aufl., Berlin 2003, Rn. 69, 97.

50 Zur präziser zu nennenden, auf das ALR zurückgehenden Doppelstruktur der Hochschulen gem. § 58 Abs. 1 S. 1 HRG, wonach die Universitäten »in der Regel Körperschaften des öffentlichen Rechts und zugleich staatliche Einrichtungen« sind, vgl. C.-C. Hsu, Institutionen körperschaftlicher Selbstverwaltung, Hamburg 2004, S. 27f.; M.E. Geis, in: Hailbronner/Geis, HRG-Kommentar (Stand: Mai 2001), § 58 Rn. 10ff.

51 Vgl. etwa § 19 Abs. 1 Nr. 10 LHG-BW; § 67 Abs. 1 Nr. 1 HSG-Bbg. Die teilweise gegebene Einschränkung, »soweit nicht gesetzlich etwas anderes bestimmt ist«, hat gegenüber dem Hamburger Modell weitgehend nur die Funktion der Etablierung einer Eilkompetenz; vgl. etwa $\S 39$ Abs.1 Nr. 1, 44 Abs. 7 HSG-SH.

52 Interessant ist auch die Genese des Gesetzes: auf eine mündliche Anfrage d. Verf., eine derartige Übertragung der Satzungsgewalt auf die Leitungsorgane sei doch ausgesprochen ungewöhnlich und seines Erachtens ohne Vorbild, antwortete der zuständige Referent in der Hamburger Behörde für Wissenschaft und Gesundheit vergnügt, man habe damals auch Zweifel gehabt, die Regelung dann aber einfach einmal in den Entwurf hineingeschrieben und abgewartet, ob die Rechtsbehörde protestiert - was sie nicht tat. Honni soit qui mal y pense.

$53 \mathrm{Zu}$ Genehmigungsvorbehalten im Hochschulrecht vgl. für die frühere Rechtslage, aber immer noch lesenswert $D$. Keller, Die staatliche Genehmigung von Rechtsakten der Selbstverwaltungsträger, Heidelberg 1976, bzgl. der Universitäten insbes. S. 140ff. 
migung von Gebührenordnungen« weder einer behördlichen Aufsichtsstelle noch dem Hochschulsenat, sondern dem neu geschaffenen Organ des Hochschulrates ${ }^{54} \mathrm{zu}$. Im Gegensatz zum Hochschulsenat handelt es sich dabei nicht um ein Vertretungsorgan der Körperschaftsmitglieder, sondern eine Schnittstelle zwischen Politik und Hochschule; die Ratsmitglieder werden gemäß $\S 84$ Abs. 3 HmbHG je zur Hälfte vom Hochschulsenat und vom Senat der Freien und Hansestadt Hamburg bestimmt, und die so bestimmten Mitglieder wählen sodann selbst ein weiteres Mitglied. Materielle Voraussetzung der Wählbarkeit ist gemäß § 84 Abs. 4 HmbHG eine gewisse Vertrautheit mit dem Hochschulwesen; die gewählten Personen sollen aus den Bereichen der Wissenschaft, Kultur, Wirtschaft oder Politik stammen, dürfen aber nicht der zuständigen Behörde angehören. Die von dem Hochschulsenat bestimmten Mitglieder dürfen jeweils zur Hälfte der Hochschule angehören. Konsequenz der Neuregelung des HmbHG ist also, daß ein Teil der autonomen Satzungserlaßbefugnis der Hochschule an ein Leitungsorgan delegiert wird, dessen Entscheidung dabei der Kontrolle durch ein weiteres, der Körperschaft gegenüber zum überwiegenden Teil heterogenes Leitungsorgan unterworfen ist. Aus dieser Warte betrachtet, handelt es sich somit nicht so sehr um einen Wechsel von hetero- zu autonomer Steuerung, sondern einen - hinsichtlich der demokratischen Legitimation fragwürdigen - Wandel in der Besetzung des Kontrollorgans.

Die angezeigte Übertragung einer partiellen Satzungsbefugnis auf ein Leitungsorgan gemäß $§ 79$ Abs. 2 S. 2 HambHG, die auf eine solche Absicherung gerade verzichtet, geht insofern noch über das aktienrechtliche Vorbild hinaus. Denn auch das Aktienrecht konzediert die spezifischen Befugnisse der Leitungsorgane primär im Hinblick auf die Entscheidungen zur Unternehmensführung im aktuellen Tagesgeschäft, darin vergleichbar dem Bürgermeister, dem die Vornahme der »Geschäfte der laufenden Verwaltung « durch die Gemeindeordnungen zur eigenverantwortlichen Wahrnehmung übertragen is ${ }^{55}$. Grundsätzlicheren Fragestellungen für die Unternehmensentwicklung gegenüber kennt dagegen auch das für das Wirtschaftsleben bestimmte Modell eine erforderliche Absicherung in Form der Entlastung von Vorstand und Aufsichtsrat durch das Repräsentativorgan Hauptversammlung ${ }^{56}$. Wenn jedoch bereits die allgemeine Übertragung dieses Modells auf den Hochschulsektor Fragen nach der Bewertung aus verfassungsrechtlicher Sicht aufwirft, weil geklärt werden muß, ob das ökonomische Paradigma der Eigenrationalität der Wissenschaft hinreichend gerecht wird ${ }^{57}$, so muß dies verstärkt für die Problematik des Satzungserlasses durch das Hochschulpräsidium gelten. In bezug auf die Einführung von Gebührensatzungen nach dem geschilderten Hamburger Modell ${ }^{58}$ müßte eine solche Diskussion al-

54 Vgl. dazu allg. T. Schmidt, Deutsche Hochschulräte. Begriff, Darstellung und rechtliche Analyse, Frankfurt a.M. 2004; ferner, allerdings mit Blick v.a. auf die Lage in Baden-Württemberg, W. Kahl, Hochschulräte - Demokratieprinzip - Selbstverwaltung, a.a.O.

$55 \mathrm{Vgl}$. E. Schmidt-Aßmann, Kommunalrecht, a.a.O., Rn. 74.

56 Gem. $§ 119$ Abs. 1 Nr. 5 AktG obliegt der Hauptversammlung ferner insbesondere das Recht zur Satzungsänderung.

57 Vgl. kritisch M.-E. Geis, Das Selbstbehauptungsrecht der Hochschulen, WissenschaftsR 37 (2004), S. 2 ff.

58 Die Regelung dürfte insofern zwischenzeitlich überholt sein, als in Hamburg Studiengebühren durch das Studienfinanzierungsgesetz vom 28.06.2006 mittlerweile gesetzlich eingeführt wurden. 
lerdings auch berücksichtigen, daß die Hochschulselbstverwaltung von vorneherein gegenständlich begrenzt ist und insbesondere keine Finanzautonomie umfaßt ${ }^{59}$.

\section{Forschungsevaluation}

Wenig beeindruckt von diesen Zweifeln transponieren die Hochschulreformen demgegenüber das ökonomische Modell konsequent nicht nur auf die inneruniversitären Verwaltungsaufgaben, sondern zumal auf die wissenschaftliche Kerntätigkeit. Auch hochschulintern sollen durch Einführung von Wettbewerbsstrukturen Effizienzsteigerungen erzielt werden, indem in der Frage der internen Finanzverteilung zunächst durch Verabschiedung von Globalhaushalten eine verstärkte Aufgabenverlagerung auf die Hochschulen vorgenommen und zugleich gesetzlich bestimmt wurde, daß diese in allen Bundesländern der Hochschul- bzw. Fakultätsleitung als Aufgabe übertragene ${ }^{60}$ Verteilung nach Maßgabe der Ergebnisse vermehrter Leistungs- und Erfolgskontrollen (unter Einbezug externer, d.h. nicht der jeweils kontrollierten Hochschule angehörender, aber ebenfalls in das Wissenschaftssystem integrierter Gutachter ${ }^{61}$ ) zu erfolgen habe ${ }^{62}$. Die sich hierbei ergebende Problematik von Methodik und Verfahren derartiger Evaluationen scheint indes noch weitgehend ungelöst ${ }^{63}$. Bedenken muß man gegen die begonnenen Reformideen aber nicht nur wegen unter Umständen ja korrigierbarer praktischer Umsetzungsschwierigkeiten haben. Gravierender scheint vielmehr, daß der gesamten Konzeption offenbar ein idealisiertes und damit in dieser Form den tatsächlichen Eigengesetzlichkeiten des Wissenschaftsbetriebs entgegenlaufendes Selbstreferenzmodell zugrunde liegt ${ }^{64}$ : Die emphatisch betonte Hochschulautonomie suggeriert, Befreiung von staatlicher Regulierung und Übertragung von Kontrollfunktionen führe automatisch zu einem Gewinn für das Wissenschaftssystem. Ignoriert wird dabei jedoch, daß hierarchische Strukturen nicht zwingend eine lähmende Wirkung entfalten müssen, sondern auch eine Entlastungsfunktion übernehmen können ${ }^{65}$. »Hinter der wohlfeilen Vokabel der Autonomie « könnte insofern auch lediglich das »aus anderen Lebensbereichen bekannte Ziel des Staates stehen, sich soweit wie möglich aus der politischen Verantwortung zurückzuziehen«, indem »der kräftezehrende Verteilungskampf « um die finanziellen Ressourcen »als moderne Form des >Divide et impera $<$ in die Universitäten verlagert wird ${ }^{66}$. Wegen seiner zwangsläufigen Verdrän-

59 Vgl. M. Fehling, Neue Herausforderungen an die Selbstverwaltung in Hochschule und Wissenschaft, a.a.O., S. 401f., der allerdings auch konstatiert, daß »vital wissenschaftsrelevant $[\ldots]$ gerade auch vieles [ist], was traditionell dem staatlichen Sektor zugerechnet wird, namentlich Finanzierungsfragen« (a.a.O., S. 408).

60 Vgl. G. Sandberger, Organisationsreformen und Autonomie, a.a.O., S. 140, 147.

61 Vgl. D. Müller-Böling, Die entfesselte Hochschule, a.a.O., S. 100; E. Hödl/W. Zegelin, Hochschulreform und Hochschulmanagement, a.a.O., S. 322ff.

62 M. Fehling, Neue Herausforderungen an die Selbstverwaltung in Hochschule und Wissenschaft, a.a.O., S. 412.

63 Vgl. den Überblick bei $H$. Seidler, in: Hailbronner/Geis, HRG-Kommentar, § 6, Rn. 10ff., v.a. $26 \mathrm{ff}$.

64 Vgl. K.F. Gärditz, Hochschulmanagement und Wissenschaftsadäquanz, a.a.O., S. 408.

65 Vgl. N. Bolz, Blindflug mit Zuschauer, München 2005, S. $39 f$.

66 M.-E. Geis, Das Selbstbehauptungsrecht der Hochschulen, a.a.O., S. 9. 
gungsfunktion tendiert der Wettbewerb zudem dazu, durch Vernachlässigung kleinerer Fächer die universitätsinterne Pluralität zu reduzieren ${ }^{67}$. Spezifisch auf die Evaluationsfragen bezogen zeigt sich eine weitere Schwierigkeit vor allem in einem unterschiedlichen Umgang mit Zeit des am ökonomischen Paradigma geschulten Wettbewerbs einer- und der wissenschaftlichen Forschung andererseits: Während diese von vorneherein in längeren Zeiträumen denkt und dabei auch Rückschläge in die eigene Verfahrensweise einkalkulieren muß, ist der Leistungsnachweis im Wettbewerb auf kurzfristige Erfolge ausgelegt. Dieser kaum leugbaren Differenz wird zwar mit der Umgehungsstrategie der Bewertung von bloßen Forschungsanträgen begegnet, die ihrerseits aber nur neue Probleme generieren. Denn jeder Antrag führt vor das Paradox, einerseits hinreichend ausführlich sein zu müssen, um eine genaue Bewertung des Vorhabens überhaupt zuzulassen, andererseits aber nicht so detailreich ausfallen zu dürfen, daß das Erfordernis weiterer finanzieller Unterstützung angesichts offenbar weitgehend bereits abgeschlossener Forschungen zu entfallen scheint. Das Zeitproblem stellt sich zudem in einer noch schlichteren Gestalt: weil die Qualifizierung von Forschungsleistungen nur durch qualifizierte Forscher erfolgen kann, müssen diese in Zukunft einen Teil ihrer Zeit für Kollegenkontrolle statt für eigene Forschungsarbeiten aufwenden. Der Hoffnung auf ein dank Evaluation stetig steigendes Niveau gründlicher wissenschaftlicher Arbeit steht damit die Befürchtung gegenüber, daß für diese einfach keine Zeit mehr bleibt. Dem »ökonomischen Paradigma entsprechend ausgedrückt: Die hohen Transaktionskosten gefährden das Ziel der optimierten Effizienz. « ${ }^{68}$ Die Erfahrung aus anderen Ländern lehrt zudem, daß unter dem Druck der Kontrolle eher die Quantität als die Qualität der Forschungsarbeiten steigen dürfte ${ }^{69}$.

Konträr zu diesen Ansätzen bleibt es dagegen denkbar, daß jenes wünschenswerte wissenschaftliche Niveau bzw. das optimale Verhältnis von Aufwand und Ertrag statt durch beharrliche Kontrolle gerade umgekehrt durch wieder vermehrte Gewährung von Freiraum ermöglicht werden könnte. Daß ein klassisches allgemeines Systemvertrauen, dem zufolge »die Sache [der Wissenschaft] ohne ihn [den Staat] unendlich besser gehen würde« und dieser »vielmehr immer hinderlich ist, sobald er sich hineinmischt $\ll^{70}$, in der Vergangenheit allzu oft enttäuscht worden sei, ist nicht ersichtlich. Dennoch nicht zu leugnende einzelne schwarze Schafe innerhalb des Wissenschafts-

67 M.-E. Geis, Das Selbstbehauptungsrecht der Hochschulen, a.a.O., S. 8.

68 M. Fehling, Neue Herausforderungen an die Selbstverwaltung in Hochschule und Wissenschaft, a.a.O., S. 414.

69 So K.-H. Ladeur, Die Wissenschaftsfreiheit der »entfesselten Hochschule«, a.a.O., S. 761, unter Berufung auf Berichte aus England; ähnlich K.F. Gärditz, Hochschulmanagement und Wissenschaftsadäquanz, a.a.O., S. 409, mit Blick auf die Schwierigkeit einer Bestimmung von Evaluationskriterien. In England sind die Erfahrungen mit dem Projekt »Research Assessment Exercise«, also der Forschungsevaluation, aufgrund der damit verbundenen Transaktionskosten so ernüchternd, daß die laufende RAE-Runde, wie C. Bode in einer anläßlich der Hundertjahrfeier des Deutschen, Englischen und Romanischen Seminars der Universität Tübingen am 15.11.2006 gehaltenen Rede herausgestellt hat, die letzte sein wird. (Der Vortrag ist bislang unveröffentlicht; für die freundliche Überlassung des Transkripts eines Tonbandmitschnittes danke ich Herrn Prof. Bode sehr herzlich.)

70 So klassisch W. v. Humboldt, Werke in fünf Bänden, Bd. IV: Schriften zur Politik und zum Bildungswesen, Darmstadt 1964, S. 257. 
betriebs blieben damit zwar ungeschoren, die offenkundige Mehrheit der seriös arbeitenden Forscher jedoch könnte die knappe Ressource Zeit der eigenen Kerntätigkeit widmen. Die Qualität ihrer Tätigkeit könnte dabei statt durch retrospektive Kontrolle stärker durch vorgelagerte prozedurale Aspekte, das heißt die Absolvierung anerkannter Qualifikationsverfahren, abgesichert werden. Nicht zuletzt liegt hierin auch ein Argument für die Beibehaltung der Habilitation als einem aussagekräftigen Kriterium zum Nachweis der Befähigung zu erfolgreicher wissenschaftlicher Arbeit.

\section{Hochschulautonomie in der Praxis}

Unter dem allgemeinen Projekt einer Verstärkung der Hochschulautonomie verbergen sich demnach, wie im Fall der Kompetenzverlagerung zugunsten der Leitungsorgane, insbesondere des Hochschulrats, de facto verringerte Einflußmöglichkeiten für die Mitglieder der Hochschulen selbst ${ }^{71}$. Die Etablierung von Netzwerkstrukturen ${ }^{72}$ zwischen Staat, Hochschulen und ihren Organen sowie intermediären Gremien wie dem Hochschulrat anstelle einer klassisch-hierarchischen Ordnung stellt zudem vor die Frage, ob Entscheidungsprozesse durch dieses Verfahren eher beschleunigt oder im Gegenteil zusätzlich verkompliziert werden ${ }^{73}$. Schließlich sieht sich das wissenschaftliche Personal der Gefahr gegenüber, durch die Übertragung spezifischer Kontrollaufgaben an seiner eigenen Forschungstätigkeit gehindert zu werden. Vor diesem Hintergrund ist äußerst fraglich, ob Anspruch und Realität der Reformvorhaben nicht deutlich auseinanderklaffen. Problematisch ist allerdings auch ihre Korrektur: Die Position des Bundesverfassungsgerichts, dem Gesetzgeber einen großen Entscheidungsspielraum zur experimentellen Fortentwicklung der überkommenen Organisationsstrukturen der Universitäten einzuräumen und diesen nur durch beigeordnete Beobachtungspflichten zu kompensieren ${ }^{74}$, könnte sich als eine Unterschätzung von irreversiblen eigendynamischen Effekten der Reformvorhaben erweisen ${ }^{75}$.

\section{Die Untätigkeitsbeschwerde}

Vergleichbare Probleme zeigen sich in einem anderen Sektor. Auch die Justiz sieht sich in jüngerer Zeit verstärkt Forderungen gegenüber, ihre Effektivität und Effizienz zu steigern. In einer Übertragung von im Verwaltungsrecht schon länger diskutierten

71 Vgl. M.-E. Geis, Das Selbstbehauptungsrecht der Hochschulen, a.a.O.

72 Vgl. dazu H.-H. Trute, Die Forschung zwischen grundrechtlicher Freiheit und staatlicher Institutionalisierung, Tübingen 1994, S. 349ff.; M. Fehling, Neue Herausforderungen an die Selbstverwaltung in Hochschule und Wissenschaft, a.a.O., S. 410f.

73 Bedenken bzgl. »Zu viel verantwortungshemmender Verflechtung « etwa bei M. Fehling, Neue Herausforderungen an die Selbstverwaltung in Hochschule und Wissenschaft, a.a.O., S. 409. Vor einem »Verwischen der Verantwortlichkeitsbereiche« warnt K.F. Gärditz, Hochschulmanagement und Wissenschaftsadäquanz, a.a.O., S. 408.

74 Vgl. BVerfGE 111, 333 (Brandenburgisches Hochschulgesetz).

75 Vgl. K.-H. Ladeur, Die Wissenschaftsfreiheit der »entfesselten Hochschule«, a.a.O., S. 755; ferner I. Augsberg/S. Augsberg, Prognostische Elemente in der Rechtsprechung des Bundesverfassungsgerichts, VerwArch 2007, S. 290 ff., v. a. S. 299 ff. 
Gedanken des »Neuen Steuerungsmodells ${ }^{76}$ finden auch hier verstärkt ökonomische Erwägungen Eingang in die Debatte ${ }^{77}$. In diesen weiteren Kontext läßt sich ein Gesetzesentwurf einordnen, den das Bundesministerium der Justiz am 26. August 2005 vorgelegt hat ${ }^{78}$ und der für alle Gerichtswege einen Rechtsbehelf gegen überlange Verfahrensdauer ${ }^{79}$ vorsieht $^{80}$. Ziel der geplanten Untätigkeitsbeschwerde ${ }^{81}$ war es, daß die Betroffenen ihr Recht auf ein zügiges Verfahren tatsächlich durchsetzen können. Die Bundesregierung begründete die Notwendigkeit der Einrichtung eines solchen Rechtsbehelfs ausdrücklich mit der neueren Rechtsprechung des EGMR ${ }^{82}$. Sowohl dieser wie das Bundesverfassungsgericht hatten in der Vergangenheit wiederholt darauf hinge-

76 Vgl. dazu allg. E. Schmidt-Aßmann, Das allgemeine Verwaltungsrecht als Ordnungsidee, a.a.O., S. 23f.; H. Hill, Neue Organisationsformen in der Staats- und Kommunalverwaltung, in: E. Schmidt-Aßmann/W. Hoffmann-Riem (Hrsg.), Verwaltungsorganisationsrecht als Steuerungsressource, Baden-Baden 1997, S. 65ff.; speziell zur Umsetzung in der Justiz M. Eifert, Das Neue Steuerungsmodell, a.a.O.; K.F. Röhl, Justiz als Wirtschaftsunternehmen, DRiZ 2000, S. 220ff.; A. Voßkuhle, Das >Produkt< der Justiz, a.a.O.

77 Vgl. dazu die Beiträge in H. Schulze-Fielitz/C. Schütz (Hrsg.), Justiz und Justizverwaltung zwischen Ökonomisierungsdruck und Unabhängigkeit, a.a.O., v.a. U. Berlit, Richterliche Unabhängigkeit und Organisation effektiven Rechtsschutzes im >ökonomisierten< Staat, a.a.O., S. 135ff.; kritisch, mit Blick auf Methodenfragen M. Reinhardt, Richterliche Unabhängigkeit im >ökonomisierten Staat<, a.a.O., S. 179ff.; ausführlich C. Schütz, Der ökonomisierte Richter. Gewaltenteilung und richterliche Unanhängigkeit als Grenzen Neuer Steuerungsmodelle in den Gerichten, Berlin 2005.

78 Der Entwurf ist abrufbar unter http://www.anwaltverein.de/01/depesche/texte05/gesetz.pdf.

79 Die durchschnittliche Verfahrensdauer variiert sowohl zwischen den Gerichtszweigen wie zwischen den einzelnen Bundesländern erheblich. Bei den Zivilgerichten dauern Verfahren in der Eingangsinstanz zwischen 3,7 und 5,8 Monaten bei den Amtsgerichten und zwischen 5,3 und 9,8 Monaten bei den Landgerichten. Die Finanzgerichte benötigen durchschnittlich 17,4 Monate zum Abschluß eines erstinstanzlichen Verfahrens. Dabei reicht die Spannbreite je nach Bundesland von 8,2 bis zu 21,7 Monaten (Zahlen zitiert nach der Pressemitteilung des Bundesjustizministeriums v. 26.08.2005, abrufbar unter www.bmj.bund.de).

80 Zum Problem vgl. K. Redeker, Kann eine Untätigkeitsbeschwerde helfen?, NJW 2003, S. 488ff.; allg. H. Sodan, Qualitätsmaßstäbe für die Justiz?, NJW 2003, S. 1494ff.; ders., Das Spannungsverhältnis von Quantität und Qualität in der Justiz, DÖV 2005, S. 764ff.

81 Das weitere Schicksal des Vorhabens ist gegenwärtig nicht genau absehbar; der Koalitionsvertrag zwischen CDU/CSU und SPD vom 11.11.2005 enthält hierzu keine Angabe, sondern spricht nur in vager Allgemeinheit davon, die Koalitionspartner würden »ein Gesamtkonzept für eine nachhaltige Sicherung der Leistungs- und Zukunftsfähigkeit der Justiz bei voller Wahrung rechtsstaatlicher Standards erstellen« (abrufbar unter http://www.bundesregierung.de/nsc_true/Content/DE/_Anlagen/koalitionsvertrag; sub VIII 2.4). Angesichts der eindeutigen Vorgaben des EGMR ist aber wohl mit einer baldigen Umsetzung zu rechnen.

82 Vgl. v.a. das Urt. v. 26.10.2000, NJW 2001, S. 2694ff.; kritisch dazu J. Meyer-Ladewig, Rechtsbehelfe gegen Verzögerungen im gerichtlichen Verfahren - zum Urteil des EGMR Kudla/Polen, NJW 2001, S. 2679ff. Früher bereits EGMR NJW 1989, S. 652; NJW 1997, S. 2809ff.; NJW 2001, S. 211; NJW 2001, S. 213. In einer erneuten jüngeren Entscheidung vom 8.6.2006, NJW 2006, S. 2389ff., bekräftigt der EGMR seine Position und ermutigt den deutschen Gesetzgeber ausdrücklich zu einer schnellen Verabschiedung eines Gesetzes mit Vorschriften, wie sie der vom BMJ vorgelegte Gesetzesentwurf enthält. Gewisse Sympathien in dieser Hinsicht ließ auch der Präsident des BVerfG, H.-J. Papier, in seinem anläßlich einer akademischen Gedächtnisfeier zu Ehren von K. A. Bettermann am 9.11.2006 an der Universität Hamburg gehaltenen Vortrag über »Staatliche Justizgewähr« erkennen. 
wiesen, daß das Recht auf eine angemessene Verfahrensdauer auch prozessual durchsetzbar sein müsse. Die Gerichte können sich danach zur Rechtfertigung einer überlangen Verfahrensdauer nicht auf die angespannte Personalsituation berufen ${ }^{83}$. Der Staat sei, so BVerfG und EGMR, vielmehr verpflichtet, alle notwendigen Maßnahmen zu treffen, damit Gerichtsverfahren in angemessener Frist beendet werden können.

Einen derartigen speziellen gesetzlichen Rechtsbehelf zum Schutz gegen eine überlange Dauer des Gerichtsverfahrens gibt es bislang jedoch noch nicht ${ }^{84}$. Die Betroffenen haben insoweit lediglich die Möglichkeit, eine Dienstaufsichtsbeschwerde gegen den Richter einzulegen oder eine - gegenüber den ordentlichen Rechtsmitteln subsidiäre und zudem unter der Einschränkung des § 93a Abs. 2 BVerfGG stehende - Verfassungsbeschwerde zu erheben. Sie können aber nicht unmittelbar auf den Fortgang des konkret anhängigen Verfahrens hinwirken. In diesen Fällen soll die neue Untätigkeitsbeschwerde Abhilfe schaffen. Nach dem vorgelegten Gesetzesentwurf muß diese Beschwerde bei dem Gericht eingelegt werden, bei dem das Verfahren anhängig ist. Dieses Gericht muß dann der Beschwerde innerhalb eines angemessenen Zeitraums abhelfen oder sie dem nächsthöheren Gericht zur Prüfung vorlegen. Will das Ausgangsgericht der Beschwerde abhelfen, so muß es innerhalb einer Frist von einem Monat nach Eingang der Beschwerde Maßnahmen zur Verfahrensförderung treffen, etwa indem es ein Gutachten in Auftrag gibt oder einen Termin für die mündliche Verhandlung bestimmt. Will es dagegen der Beschwerde nicht abhelfen, entscheidet das nächsthöhere Gericht abschließend über die Untätigkeitsbeschwerde. Hält dieses die Beschwerde für begründet, kann es dem Ausgangsgericht eine Frist setzen, innerhalb derer wirksame Maßnahmen zur Verfahrensförderung ergriffen werden müssen.

Daß dieses Vorgehen, dem zur Entscheidung berufenen Gericht konkrete Vorgaben hinsichtlich der Prozeßförderung aufzugeben, in bezug auf die richterliche Unabhängigkeit gemäß Art. 97 Abs. 1 GG, die u.a. die Unabhängigkeit gegenüber der Rechtsauffassung der Obergerichte umfaßt ${ }^{85}$, jedenfalls nicht gänzlich unproblematisch ist, dürfte klar $\operatorname{sein}^{86}$. Zweifelhaft ist aber vor allem, ob mit der Einführung einer solchen Beschwerde der Zweck der effektiven Verfahrensbeschleunigung erreicht werden kann. Schon der Richter J. Casadevall hat in einer dissenting opinion zu dem Urteil des EGMR vom 26. Oktober 2000 erhebliche diesbezügliche Bedenken geltend gemacht ${ }^{87}$.

83 Grundlegend BVerfGE 36, 264 (275); daran ausdrücklich anschließend zuletzt sehr deutlich BVerfG, Beschl. v. 29.11.2005, 2 BvR 1737/05, NJW 2006, S. 668ff.

84 Richterrechtlich ist eine Untätigkeitsbeschwerde dagegen als außerordentlicher Rechtsbehelf mit dem Zweck, den Anspruch der Verfahrensbeteiligten auf einen effektiven Rechtschutz zu gewährleisten, etwa im Zivilrecht geschaffen worden. Vgl. dazu OLG Karlsruhe, FamRZ 2004, S. 53 (54); OLG Dresden, FamRZ 2000, S. 1422f.; OLG Saarbrücken, NJWRR 1999, S. 1290f.; ferner P. Gummer, in: Zöller, ZPO, 24. Auflage, § 567 Rn. 21; K. Reichhold, in: Thomas/Putzo, ZPO, 26. Auflage, § 567 Rn. 10.

85 BVerfGE 87, 273 (278).

86 Vgl. K. Redeker, Kann eine Untätigkeitsbeschwerde helfen?, a.a.O., S. 489, mit dem berechtigten Hinweis, daß die bloße Fristsetzung durch das Beschwerdegericht die Unabhängigkeit allerdings noch nicht verletze.

87 Vgl. J. Casadevall, NJW 2001, S. 2701f.; dazu auch K. Redeker, Kann eine Untätigkeitsbeschwerde helfen?, a.a.O., S. 489ff., der im Ergebnis einen Nutzen der Untätigkeitsbeschwerde aber dennoch bejahen will. 
Tatsächlich muß man davon ausgehen, daß eine solche Beschwerde insbesondere dann Erfolg haben kann, wenn die Verfahrensverzögerung keine objektiven, sondern primär subjektive Ursachen hat: also Faulheit statt Überlastung. Denn nur in diesem Fall wäre das Versagen nicht systembedingt, sondern als vom einzelnen Richter verursacht auch von ihm korrigierbar. Geht man dagegen davon aus, daß die jedenfalls weit überwiegende Mehrzahl der zu Recht als überlang gescholtenen Verfahrensdauern nicht auf persönliche Defizite der Entscheidungsträger, sondern objektive Mißstände in der Gerichtsorganisation zurückgeht, wird eine - vom Gesetzesentwurf erstaunlich freimütig eingeräumte $^{88}$ - zusätzliche Belastung durch die Einführung eines weiteren Rechtsmittels die Situation kaum verbessern helfen ${ }^{89}$. Was im einen Extremfall zu einer gewissen Beschleunigung führen mag, dürfte an anderen, weniger offensichtlichen Stellen eher weitere Verzögerungen produzieren. Insofern ist hier wie bezüglich der Forschungsevaluationen zu fragen, ob im gesamtsystemischen Saldo sich nicht das Hinnehmen einzelnen Fehlverhaltens als letztlich immer noch produktiver erweisen könnte denn eine alle Systemhandlungen - als Subjekt oder Objekt - jedenfalls virtuell (ein entsprechendes anwaltliches Taktieren dürfte auch in sachlich wenig begründeten Fällen denkoder sogar erwartbar sein) belastende Überwachungstätigkeit.

Der Glaube an den effektiven Nutzen der Einführung einer Untätigkeitsbeschwerde muß dagegen mit der Unterstellung operieren, daß die gegenwärtig zu beobachtenden Verzögerungen zu einem zumindest erheblichen Teil das Ergebnis richterlichen Fehlverhaltens sind. Diese Prämisse sollte man zumindest offenlegen. Empirische Nachweise ihrer Begründetheit wurden im Entwurf nicht vorgelegt.

\section{Regulierte Selbstregulierung reloaded?}

Die Effektivität einer verstärkten Aufgabenverlagerung auf die sozialen Subsysteme kann demnach mit guten Gründen in Zweifel gezogen werden. In wieder stärker theoretisch orientierter Hinsicht bleibt damit allerdings noch zu klären, inwieweit aufgrund der dargelegten Dysfunktionalitäten auch die Grundkonzeption der regulierten Selbstregulierung aufzugeben bzw. jedenfalls zu modifizieren ist. Zwei Fragen legen sich nahe: Handelt es sich, zum einen, bei den gezeigten Beispielen überhaupt um systemtheoretisch inspirierte Konzepte? Ist, anders formuliert, das Scheitern aus theorieimmanenten Gründen unabdingbar? Und falls nicht: Welche Folgerungen müßten dann, zum anderen, aus der theoretischen Orientierung einer- und den praktischen Umsetzungsproblemen andererseits für eine Neudimensionierung des Selbstregulierungskonzeptes gezogen werden? Es geht mithin nicht um eine - ohnehin kaum zu leistende

88 Unter dem Punkt »D. Finanzielle Auswirkungen auf die öffentlichen Haushalte« heißt es insofern: »Da mit dem Gesetzgebungsvorhaben die Möglichkeit einer Untätigkeitsbeschwerde eingeführt wird, kann sich das Aufkommen an Verfahren sowohl bei den Instanzgerichten als auch bei den obersten Bundesgerichten erhöhen. Diese lassen sich jedoch mit den vorhandenen Personalkapazitäten erfüllen.«

89 In diese Richtung zielte - erwartbarerweise - auch die Stellungnahme des Deutschen Richterbundes zum Gesetzesvorschlag (abrufbar unter: http://www.drb.de/pages/html/stellung/ st-untaetigkeit.html). 
- Motivforschung bezüglich der subjektiven Vorstellung der Gesetzesautoren, sondern die theoretische Nachkonstruktion des objektiv Geleisteten.

\section{Recht als Immunsystem der Gesellschaft}

Als Antwort auf die erste Frage kann man mit dem Autor der »Sozialen Systeme« ein vorsichtiges »Nein « riskieren. Denn für Luhmann war klar, daß die »Umstellung auf Autonomie des Rechtssystems ${ }^{90}{ }$, d.h. seine operative Geschlossenheit, eine spezifische Funktionsbeschreibung beinhaltete. Ausdifferenzierung bedeutet insofern - gewissermaßen als systemtheoretische Aufarbeitung des Gedankens der arbeitsteiligen Gesellschaft $^{91}$ - auch Aufgabenteilung. Durch sie wird einem gesellschaftlichen Teilbereich die Rolle des innersozialen Immunsystems, das Exzesse von Teilen zugunsten des Ganzen in Grenzen halten und etwaige Totalisierungsbestrebungen zurückhalten sollte, zugeschrieben: dem Recht ${ }^{92}$. Die Autoreferentialität der ausdifferenzierten Subsysteme meint in diesem Sinne gerade nicht, daß jeweils innersystemisch eine Art Mini-Polizei installiert wird, die die eigenen Prozesse auf ihre Verträglichkeit mit dem Gesellschaftsganzen überwacht. Vielmehr wird durch eine solche Zusatzfunktion die mit der Ausdifferenzierung einhergehende Spezialisierung wieder unterlaufen. In der durch die funktionale Differenzierung erreichten Ordnung »kann kein Funktionssystem ein anderes ersetzen oder auch nur in seiner Funktion entlasten: Weder kann die Politik anstelle der Wirtschaft handeln noch die Wirtschaft anstelle der Wissenschaft forschen. Weder kann die Wissenschaft für das Recht einspringen noch das Recht für die Religion - und so fort in allen Intersystembeziehungen. Gerade dieser Verzicht auf Multifunktionalität, auf Mehrfachsicherung der Funktionen, findet in der Autonomie der Funktionssysteme Ausdruck « ${ }^{93}$. Wenn das Konzept der Selbstregulierung funktionieren soll, dann demnach nur unter der Prämisse, daß weder systemfremde Aufgaben übertragen noch auch eine interne Überlastung mit zwar systemkonformen, aber externen Zwecken dienenden Aufgaben erfolgt. Der »Schluß von der Systemtheorie auf die Regelungstechnik « im Sinne der gekennzeichneten Selbstregulierungsstrategien enthält damit nicht nur einen Kategorienfehler, weil auch die »Reflexion von Steuerungs-

90 N. Luhmann, Das Recht der Gesellschaft, a.a.O., S. 62. Luhmann läßt zwar die Möglichkeit ausdrücklich offen, daß sich dieser Prozeß auch verändern (»Anders als in der Parsonschen Theorie des allgemeinen Handlungssystems sehen wir funktionale Differenzierung als ein evolutionäres Produkt und nicht als eine logische Folge der Analyse des Handlungsbegriffs. «) und sich insofern die Autonomsetzung des Rechts als »europäische Anomalie, die sich in der Evolution einer Weltgesellschaft abschwächen wird « (Das Recht der Gesellschaft, a.a.O., S. 585f.) zeigen könnte. Funktionale Differenzierung und autonomes Recht bleiben damit aber verkoppelt.

91 Vgl. zum Problem allg. S. Holmes, Differenzierung und Arbeitsteilung im Denken des Liberalismus, in: N. Luhmann (Hrsg.), Soziale Differenzierung, a.a.O., S. 9ff.

92 Vgl. N. Luhmann, Soziale Systeme, a.a.O., S. 504ff., v.a. 509ff.; ders., Das Recht der Gesellschaft, a.a.O., S. 565ff.; mit besonderem Bezug auf die Aufgabe der Grundrechte als Entdifferenzierungssperre ferner ders., Grundrechte als Institution. Ein Beitrag zur politischen Soziologie, 2. Aufl. Berlin 1975.

93 N. Luhmann, Die soziologische Beobachtung des Rechts, Frankfurt a.M. 1986, S. 13. 
mängeln ein Internum des Rechtssystems « bleibt ${ }^{94}$. Ein gewisses Maß an imperativisch verfahrender Steuerung ist vielmehr in der Ausdifferenzierung selbst angelegt. Gerade die nicht länger stratifikatorisch, sondern funktional differenzierte Gesellschaft kann zur Komplexitätsbewältigung auf die entlastenden Effekte von Hierarchisierung und externer Kontrolle nicht verzichten ${ }^{95}$. Entsprechend ist ein Recht, das seine primäre Aufgabe nur noch in der Gestaltung allgemeiner Zweckprogramme begreift, welche ihrerseits dann in den einzelnen Sozialbereichen allererst noch konkretisiert werden müssen, für Luhmann unvorstellbar. Ihm zufolge bildet vielmehr die konditionale Programmierung ein unabdingbares Proprium des Rechtssystems, um die systemintern zu berücksichtigenden Tatsachen hinreichend zu limitieren ${ }^{96}$ - und das heißt vor allem, um sie durch die in ihrer besonderen Bedeutung als Zentrum des Systems hervorgehobenen ${ }^{97}$ kontrollierenden Gerichte justitiabel zu halten. Die Theorie der operativen Geschlossenheit gesellschaftlicher Teilsysteme wird insofern »durch Konzepte gesellschaftlicher Selbststeuerung eher in Frage gestellt. ${ }^{98}$

\section{Effektive Selbstregulierung}

Damit ist zugleich der Anhalt für die Beantwortung der zweiten Frage gewonnen. Das Konzept der regulierten Selbstregulierung darf nicht dazu führen, zwar prima facie die Ausdifferenzierung der Gesellschaft zu unterstützen, in den tatsächlichen Konsequenzen jedoch eher einzuschränken. Diese Gefahr besteht einerseits für das Recht selbst und drängt insofern legitimerweise auf zumindest partielle Delegation rechtlicher Kontrollfunktionen ${ }^{99}$. Sie besteht jedoch zumal überall dort, wo die Ersetzung der rechtlichen Regelungsmechanismen nicht mit großer Vorsicht auf inkompatible Eigenrationalitäten der verschiedenen Subsysteme achtet, sondern statt dessen undifferenziert die Steuerungsvorstellungen eines einzelnen Sozialbereichs in anderen Gebieten zur Anwendung kommen läßt. Wenn und soweit die Autonomie der einzelnen Sphären gewährt und gestärkt werden soll, muß die an die Stelle des Rechts tretende Handlungslogik dem speziellen Regelungsbereich entsprechen. Gerade eine konsequente ökonomische Analyse müßte insofern zu einer Selbstbeschränkung des wirtschaftlichen Paradigmas führen,

94 Vgl. Chr. Möllers, Braucht das öffentliche Recht einen neuen Methoden- und Richtungsstreit?, a.a.O., S. 205.

95 Vgl. N. Bolz, Blindflug mit Zuschauer, a.a.O., S. 39f., 176f.

96 Vgl. N. Luhmann, Das Recht der Gesellschaft, a.a.O., S. 195.

97 Vgl. N. Luhmann, Das Recht der Gesellschaft, a.a.O., S. 297ff., besonders S. 320ff.

98 Chr. Möllers, Braucht das öffentliche Recht einen neuen Methoden- und Richtungsstreit?, a.a.O., S. 205, mit Verweis auf R. Mayntz, Politische Steuerung und gesellschaftliche Steuerungsprobleme - Anmerkungen zu einem theoretischen Paradigma, JbStVW 1 (1987), S. 89ff, hier S. 100ff. Die Protagonisten der Steuerungsdiskussion befinden sich, aus dieser Perspektive betrachtet, nicht mehr innerhalb, sondern bereits jenseits des Luhmannschen Modells. A fortiori muß das für die neueren Debatten zum Governance-Konzept und den ihm korrespondierenden Regelungsstrukturen (vgl. dazu etwa den Überblick bei C. Franzius, Governance und Regelungsstrukturen, VerwArch 2006, S. 186ff.) gelten.

99 Zum Problem vgl. G. Teubner, Ein Fall von struktureller Korruption? Die Familienbürgschaft in der Kollision unverträglicher Handlungslogiken, KritV 83 (2000), S. 388ff. 
weil die undifferenzierte Oktroyierung des wirtschaftlichen Kalküls zu zahlreichen Friktionen mit den hiermit im Kern unvereinbaren besonderen Handlungsrationalitäten etwa von Wissenschaft und Recht führen und damit letztlich Effizienz und Effektivität dieser Sozialbereiche eher lähmen denn steigern dürfte. Das schließt eine durch auch ökonomische Erwägungen angeleitete Supervision nicht aus, weist aber zugleich darauf hin, wo diese durch dysfunktionale side-effects sich selbst zu konterkarieren droht. Ein effizienzorientiertes Modell regulierter Selbstregulierung muß demnach zweierlei leisten: es muß zum einen, will es mehr sein als eine bloß zusätzliche Belastung, rechtliche Konfliktlösungsmechanismen und Regelungsstrategien nicht lediglich supplementieren, sondern tatsächlich substituieren. Und es muß dabei zum anderen den immanenten Gegebenheiten des jeweiligen Regelungsbereichs Rechnung tragen. Die damit gestellte Aufgabe ist ebenso komplex wie anspruchsvoll. Daß sie dennoch nicht gänzlich unbewältigbar sein könnte, zeigen jedoch einige recht vielversprechend erscheinende Ansätze in bezug auf die Konfliktlösung hinsichtlich von Verletzungen des Persönlichkeitsrechts im Bereich der sogenannten Unterhaltungsöffentlichkeit ${ }^{100}$.

\section{Schlu $\beta$}

Auch im rechtswissenschaftlichen Kontext droht die kurzschlüssige Folgerung »modern und also systemtheoretisch ${ }^{101}$ zum allzu geläufigen, kaum noch nachweisbedürftigen Glaubensartikel zu werden. Konträr hierzu muß die Jurisprudenz indes auf eine paradoxe Weise gerade dann, wenn sie die These von der autopoietischen Geschlossenheit der gesellschaftlichen Subsysteme ernst nimmt, gegenüber dem Einbau soziologischer Theorieelemente in die Rechtsdogmatik große Vorsicht walten lassen. Nicht zuletzt Luhmann selbst war sich dieser zu Recht als »Problem der Interdisziplinarität ${ }^{102}$ bezeichneten Schwierigkeit sehr bewußt ${ }^{103}$. Die Kritik an dem systemtheoretisch inspirierten Modell der regulierten Selbstregulierung ${ }^{104}$ ist demnach im Ansatz

100 Vgl. K.-H. Ladeur, Helmut Ridders Konzeption der Meinungs- und Pressefreiheit in der Demokratie, KJ 1999, S. 281ff.; ders., Die Anpassung des privaten Medienrechts an die »Unterhaltungsöffentlichkeit«, NJW 2004, S. 393ff.; daran anknüpfend A. Gosche, Das Spannungsverhältnis zwischen Meinungsfreiheit und Ehrenschutz in der fragmentierten Öffentlichkeit, Baden-Baden 2007. Zumindest ein Ansatz zur Differenzierung findet sich auch in BVerfG, NJW 1998, S. 1386 (Koschwitz/»Münzenerna«).

101 J. Hörisch, Ende der Vorstellung. Die Poesie der Medien, Frankfurt a.M. 1999, S. 61.

102 O. Lepsius, Steuerungsdiskussion, Systemtheorie und Parlamentarismuskritik, a.a.O., S. 7.

103 In seinen späteren Schriften hat er einen dogmatischen Nutzen der soziologischen Beobachtungen eher in Abrede gestellt. Vgl. N. Luhmann, Die soziologische Beobachtung des Rechts, a.a.O., S. 44: »Ein Nutzen für die Rechtspraxis ist von Soziologie kaum zu erwarten.« Angesichts der konkreten dogmatischen Folgerungen aus der Theorie, wie sie in »Grundrechte als Institution« (a.a.O.) noch demonstriert wurden, kann allerdings fraglich erscheinen, ob diese negationistische Haltung nicht ihrerseits wiederum zu vorsichtig war. Vgl. insofern auch etwas offener ders., Das Recht der Gesellschaft, a.a.O., S. 24: »Anwendungen [der Systemtheorie im juristischen Bereich] sollen damit nicht ausgeschlossen sein, aber sie werden sich mehr sporadisch und punktuell, eher zufällig und eher in der Form von Irritationen als in der Form logischer Schlüsse ergeben.«

104 Vgl. O. Lepsius, Steuerungsdiskussion, Systemtheorie und Parlamentarismuskritik, a.a.O. 
berechtigt und verdient nähere Beachtung. Wenn ihr dennoch in der bislang vorgetragenen Form nicht vorbehaltlos zugestimmt werden kann, so deshalb, weil sie nicht nur im Nachvollzug der systemtheoretischen Implikationen, sondern zumal in der Analyse der im einzelnen vorgeschlagenen Konzepte zu kurz greift und statt dessen zu früh allein auf die demokratietheoretische Problematik verweist. Eine solche nähere Analyse dürfte das Konzept der Selbstregulierung als vielschichtiger erweisen, als die Kritik an diesem Vorgehen zugeben mag. Unter dem einheitlichen Titel verbergen sich nämlich durchaus unterschiedliche, ein teilweise stark divergierendes Staatsverständnis voraussetzende Konzeptionen ${ }^{105}$. Erst im Rahmen einer differenzierteren Auseinandersetzung mit diesen dem allgemeinen Projekt immanenten divergenten Tendenzen könnte über das Modell der regulierten Selbstregulierung und die in diesem Zusammenhang relevante Valenz systemtheoretischen Gedankensguts für die Rechtswissenschaft und die praktischen Ergebnisse der Steuerungsdiskussion ein abschließendes Urteil gesprochen werden.

105 Exemplarisch dafür können etwa die bei aller Übereinstimmung in der grundsätzlichen innovationsfreundlichen Richtung dennoch hinreichend Diskrepanzen aufweisenden Ansätze von Wolfgang Hoffmann-Riem einer- und Karl-Heinz Ladeur andererseits genannt werden. Claudio Franzius (Governance und Regelungsstrukturen, a.a.O., S. 215, Anm. 165) spricht insofern sogar von »entgegengesetzten Grundannahmen « bezüglich des Vertrauens in gesellschaftliche Problemlösungen. Vgl. zu den Positionen etwa W. HoffmannRiem, Modernisierung von Recht und Justiz. Eine Herausforderung des Gewährleistungsstaates. Frankfurt/M. 2001; K.-H. Ladeur, Der Staat gegen die Gesellschaft. Zur Verteidigung der Rationalität der »Privatrechtsgesellschaft«, Tübingen 2006. 